\title{
Bank Crashes and Micro Enterprise Loans
}

\author{
Marco Desogus \\ $\mathrm{PhD}$ in Economics \\ Economist and Credit Financial Advisor \\ Italy \\ Beatrice Venturi \\ Associate Professor \\ Mathematics for Economics and Social Science \\ Dept. of Economics and Business Science \\ University of Cagliari \\ Italy
}

\begin{abstract}
This paper begins with an analysis of trends - over the period 2012-2018 - for total bank loans, non-performing loans and the number of active, working enterprises. A review survey was done on national data from Italy with a comparison developed on a local subset from the Sardinian Region. Empirical evidence appears to support the hypothesis of the paper: can the rating class assigned by banks - using current IRB and A-IRB systems - to micro and very small enterprises, whose ability to replace financial resources using endogenous means is structurally impaired, ipso facto orient the results of performance in the same terms of PD-Probability of Default assigned by the algorithm, thereby upending the principle of cause and effect? The thesis is developed through mathematical modelling that demonstrates the interaction of the measurement tool (the rating algorithm applied by banks) on the collapse of the loan status (default, performing or some intermediate point) of the assessed micro-entity. Emphasis is given, in conclusion, to the phenomenon using evidence of the intrinsically mutualistic link of the two populations of banks and (micro) enterprises provided by a system of differential equations.
\end{abstract}

Keywords: credit big data, rating models, MSE (Micro Small Enterprises) lending, financial system stability

JEL Codes: C02; C18; G24

\section{Introduction: empirical evidence}

Firstly, trend data (for the years 2012-2018) was collected and organised on the number of enterprises in the Sardinian regional and Italian national production sectors and, over the same interval, on their performance trends from the credit and financial system.Necessarily, a selection of the graphs, with which the analyses was observed, that provide reasoning for this workare included below.

Section figures 1 and 2 - Number of micro enterprises (normalization from ISTAT and Chambers of Commerce sources ${ }^{1}$ ) 


\section{Figure 1}

\begin{tabular}{|c|c|c|c|c|c|c|c|}
\hline Year & 2012 & 2013 & 2014 & 2015 & 2016 & 2017 & 2018 \\
\hline $\begin{array}{l}\text { B: extraction of minerals from quarries } \\
\text { and mines }\end{array}$ & 117 & 112 & 97 & 93 & 94 & 102 & 102 \\
\hline C: manufacturing & 7,492 & 7,283 & 6,886 & 6,814 & 6,828 & 7,023 & 7,031 \\
\hline $\begin{array}{l}\text { D: supply of electricity, gas, steam and air } \\
\text { conditioning }\end{array}$ & 85 & 109 & 107 & 113 & 132 & 109 & 109 \\
\hline $\begin{array}{l}\text { E: supply of water, sewerage, waste } \\
\text { management and environmental } \\
\text { remediation services }\end{array}$ & 190 & 198 & 199 & 214 & 207 & 201 & 201 \\
\hline F: construction & 14,340 & 13,773 & 13,121 & 12,619 & 12,639 & 13,227 & 13,243 \\
\hline $\begin{array}{l}\text { G: wholesale and retail trade, repair of } \\
\text { motor vehicles and motorcycles }\end{array}$ & 30,252 & 30,137 & 29,205 & 28,653 & 28,993 & 29,290 & 29,326 \\
\hline H: transport and storage & 3,090 & 3,038 & 2,910 & 2,807 & 2,853 & 2,924 & 2,927 \\
\hline $\begin{array}{l}\text { I: accommodation and food service } \\
\text { businesses }\end{array}$ & 9,409 & 9,521 & 9,424 & 9,499 & 9,795 & 9,478 & 9,490 \\
\hline $\begin{array}{l}\mathrm{J}: \text { information and communications } \\
\text { services }\end{array}$ & 1,856 & 1,835 & 1,783 & 1,801 & 1,821 & 1,809 & 1,812 \\
\hline $\begin{array}{l}\text { K: financial and insurance service } \\
\text { businesses }\end{array}$ & 1,613 & 1,612 & 1,634 & 1,637 & 1,668 & 1,624 & 1,626 \\
\hline L: real estate businesses & 2,776 & 2,946 & 2,860 & 2,863 & 3,016 & 2,877 & 2,880 \\
\hline $\begin{array}{l}\text { M: professional, scientific and technical } \\
\text { businesses }\end{array}$ & 15,880 & 15,445 & 15,375 & 15,552 & 15,880 & 15,542 & 15,562 \\
\hline $\begin{array}{l}\text { N: rental and travel agencies, business } \\
\text { support services }\end{array}$ & 3,313 & 3,165 & 3,135 & 3,141 & 3,187 & 3,171 & 3,175 \\
\hline P: education & 528 & 537 & 532 & 533 & 535 & 530 & 531 \\
\hline Q: healthcare and social services & 6,161 & 6,218 & 6,422 & 6,612 & 6,713 & 6,391 & 6,399 \\
\hline $\begin{array}{l}\text { R: arts, sports, entertainment and } \\
\text { amusement businesses }\end{array}$ & 1,217 & 1,193 & 1,178 & 1,146 & 1,207 & 1,182 & 1,183 \\
\hline S: other service businesses & 4,528 & 4,552 & 4,536 & 4,604 & 4,737 & 4,567 & 4,572 \\
\hline TOTAL & 102,847 & 101,674 & 99,404 & 98,701 & 100,305 & 100,045 & 100,169 \\
\hline
\end{tabular}

Figure 2

\begin{tabular}{|l|l|l|l|l|l|l|l|}
\hline $\begin{array}{l}\text { years/number of micro enterprises } \\
\text { in Sardinia vectors }\end{array}$ & 2012 & 2013 & 2014 & 2015 & 2016 & 2017 & 2018 \\
\cline { 1 - 7 } & 102,847 & 101,674 & 99,404 & 98,701 & 100,305 & 100,045 & 100,169 \\
\hline
\end{tabular}




\section{Sardinia - number of micro enterprises}

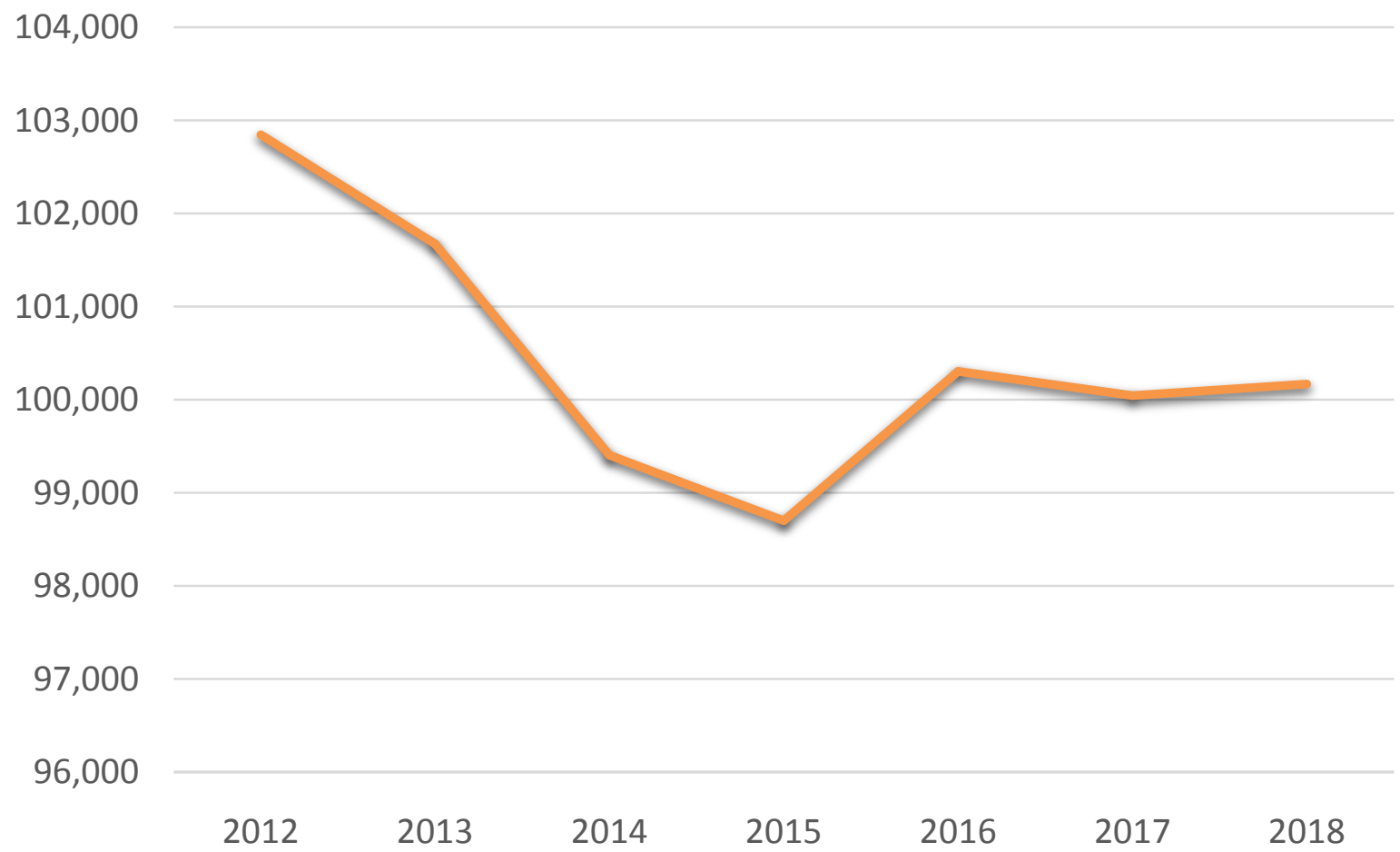

\begin{tabular}{|c|c|c|c|c|c|c|c|}
\hline$\underline{\text { Year }}$ & 2012 & 2013 & 2014 & 2015 & 2016 & 2017 & 2018 \\
\hline $\begin{array}{l}\text { B: extraction of minerals from } \\
\text { quarries and mines }\end{array}$ & 1,907 & 1,850 & 1,775 & 1,712 & 1,796 & 1,795 & 1,795 \\
\hline C: manufacturing & 345,293 & 338,015 & 328,486 & 321,837 & 330,613 & 330,526 & 330,459 \\
\hline $\begin{array}{l}\text { D: supply of electricity, gas, } \\
\text { steam and air conditioning }\end{array}$ & 8,380 & 9,610 & 9,916 & 10,205 & 9,448 & 9,445 & 9,443 \\
\hline $\begin{array}{l}\text { E: supply of water, sewerage, } \\
\text { waste management and } \\
\text { environmental remediation } \\
\text { services }\end{array}$ & 6,485 & 6,688 & 6,748 & 6,816 & 6,628 & 6,626 & 6,625 \\
\hline F: construction & 548,709 & 528,592 & 509,648 & 492,388 & 515,477 & 515,341 & 515,237 \\
\hline $\begin{array}{l}\text { G: wholesale and retail trade, } \\
\text { repair of motor vehicles and } \\
\text { motorcycles }\end{array}$ & $1,124,546$ & $1,116,087$ & $1,086,631$ & $1,068,659$ & $1,089,768$ & $1,089,481$ & $1,089,262$ \\
\hline H: transport and storage & 119,126 & 117,430 & 113,241 & 110,756 & 114,173 & 114,143 & 114,120 \\
\hline $\begin{array}{l}\text { I: accommodation and food } \\
\text { service businesses }\end{array}$ & 288,119 & 294,007 & 292,996 & 295,706 & 290,253 & 290,177 & 290,119 \\
\hline $\begin{array}{lcc}\mathrm{J}: & \text { information } & \text { and } \\
\text { communications services }\end{array}$ & 91,274 & 89,895 & 91,020 & 92,279 & 90,353 & 90,329 & 90,311 \\
\hline
\end{tabular}




\begin{tabular}{|c|c|c|c|c|c|c|c|}
\hline $\begin{array}{l}\text { K: financial and insurance } \\
\text { service businesses }\end{array}$ & 88,998 & 90,637 & 92,831 & 93,799 & 90,799 & 90,775 & 90,757 \\
\hline L: real estate businesses & 234,738 & 242,874 & 238,492 & 237,637 & 236,437 & 236,374 & 236,327 \\
\hline $\begin{array}{l}\text { M: professional, scientific and } \\
\text { technical businesses }\end{array}$ & 702,053 & 683,778 & 698,154 & 707,020 & 691,902 & 691,720 & 691,581 \\
\hline $\begin{array}{l}\text { N: rental and travel agencies, } \\
\text { business support services }\end{array}$ & 132,452 & 128,082 & 128,721 & 128,394 & 128,327 & 128,294 & 128,268 \\
\hline P: education & 25,239 & 25,957 & 27,351 & 27,781 & 26,359 & 26,352 & 26,347 \\
\hline $\begin{array}{l}\text { Q: healthcare and social } \\
\text { services }\end{array}$ & 253,160 & 254,655 & 270,894 & 278,646 & 262,123 & 262,054 & 262,001 \\
\hline $\begin{array}{l}\text { R: arts, sports, entertainment } \\
\text { and amusement businesses }\end{array}$ & 60,658 & 60,382 & 62,001 & 63,011 & 60,997 & 60,981 & 60,969 \\
\hline S: other service businesses & 198,593 & 196,542 & 199,755 & 200,185 & 197,103 & 197,051 & 197,011 \\
\hline TOTAL & $4,229,730$ & $4,185,081$ & $4,158,660$ & $4,136,831$ & $4,142,556$ & $4,141,465$ & $4,140,633$ \\
\hline
\end{tabular}

Figure 4

\begin{tabular}{|l|l|l|l|l|l|l|l|}
\hline & 2012 & 2013 & 2014 & 2015 & 2016 & 2017 & 2018 \\
\hline $\begin{array}{l}\text { years/number of micro } \\
\text { enterprises in Italy vectors }\end{array}$ & $4,229,730$ & $4,185,081$ & $4,158,660$ & $4,136,831$ & $4,142,556$ & $4,141,465$ & $4,140,633$ \\
\hline
\end{tabular}

\section{Italy - number of micro enterprises}

$$
\begin{array}{r}
4,240,000 \\
4,220,000 \\
4,200,000 \\
4,180,000 \\
4,160,000 \\
4,140,000 \\
4,120,000 \\
4,100,000 \\
4,080,000
\end{array}
$$

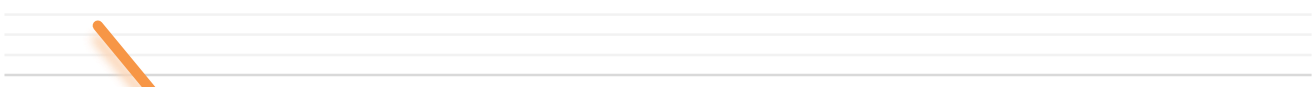

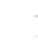

$-$




\section{Figure 5}

\begin{tabular}{|c|c|c|c|c|c|c|c|}
\hline Year & 2012 & 2013 & 2014 & 2015 & 2016 & 2017 & 2018 \\
\hline $\begin{array}{l}\text { B: extraction of minerals from quarries } \\
\text { and mines }\end{array}$ & 35 & 32 & 30 & 30 & 28 & 33 & 34 \\
\hline C: manufacturing & 658 & 607 & 566 & 521 & 537 & 619 & 625 \\
\hline $\begin{array}{l}\text { D: supply of electricity, gas, steam and } \\
\text { air conditioning }\end{array}$ & 12 & 14 & 12 & 11 & 11 & 13 & 13 \\
\hline $\begin{array}{l}\text { E: supply of water, sewerage, waste } \\
\text { management and environmental } \\
\text { remediation services }\end{array}$ & 96 & 95 & 84 & 87 & 80 & 95 & 96 \\
\hline F: construction & 514 & 448 & 375 & 365 & 371 & 444 & 448 \\
\hline $\begin{array}{l}\text { G: wholesale and retail trade, repair of } \\
\text { motor vehicles and motorcycles }\end{array}$ & 792 & 774 & 712 & 712 & 729 & 796 & 805 \\
\hline H: transport and storage & 266 & 259 & 251 & 254 & 274 & 279 & 282 \\
\hline $\begin{array}{l}\text { I: accommodation and food service } \\
\text { businesses }\end{array}$ & 469 & 437 & 438 & 460 & 527 & 499 & 504 \\
\hline $\begin{array}{l}\mathrm{J}: \text { information and communications } \\
\text { services }\end{array}$ & 67 & 69 & 76 & 73 & 68 & 76 & 76 \\
\hline $\begin{array}{l}\text { K: financial and insurance service } \\
\text { businesses }\end{array}$ & 29 & 31 & 27 & 25 & 31 & 31 & 31 \\
\hline L: real estate businesses & 19 & 12 & 7 & 9 & 7 & 12 & 12 \\
\hline $\begin{array}{l}\text { M: professional, scientific and technical } \\
\text { businesses }\end{array}$ & 79 & 78 & 74 & 74 & 84 & 83 & 84 \\
\hline $\begin{array}{l}\text { N: rental and travel agencies, business } \\
\text { support services }\end{array}$ & 259 & 244 & 250 & 233 & 251 & 265 & 268 \\
\hline P: education & 31 & 29 & 33 & 34 & 38 & 35 & 36 \\
\hline Q: healthcare and social services & 284 & 294 & 302 & 301 & 318 & 321 & 324 \\
\hline $\begin{array}{l}\text { R: arts, sports, entertainment and } \\
\text { amusement businesses }\end{array}$ & 69 & 62 & 62 & 62 & 71 & 70 & 71 \\
\hline S: other service businesses & 72 & 72 & 71 & 65 & 72 & 75 & 76 \\
\hline TOTAL & $3, \mathbf{3 5 1}$ & 3 & 3 & 3,316 & $3, \mathbf{3 , 4 9 7}$ & $3,3,746$ & $3,3,784$ \\
\hline
\end{tabular}

Figure 6

\begin{tabular}{|l|l|l|l|l|l|l|l|}
\hline $\begin{array}{l}\text { years/number of Macro enterprises in } \\
\text { Sardinia vectors }\end{array}$ & 2012 & 2013 & 2014 & 2015 & 2016 & 2017 & 2018 \\
\hline & 3,751 & 3,557 & 3,370 & 3,316 & 3,497 & 3,746 & 3,784 \\
\hline
\end{tabular}




\section{Sardinia - number of macro enterprises}

3,900
3,800
3,700
3,600
3,500
3,400
3,300
3,200
3,100
3,000

$\begin{array}{lllllll}2012 & 2013 & 2014 & 2015 & 2016 & 2017 & 2018\end{array}$

Figure 7

\begin{tabular}{|c|c|c|c|c|c|c|c|}
\hline$\underline{\text { Year }}$ & 2012 & 2013 & 2014 & 2015 & 2016 & 2017 & 2018 \\
\hline B: extraction of minerals from quarries and mines & 544 & 486 & 482 & 474 & 454 & 522 & 537 \\
\hline C: manufacturing & 72,013 & 69,329 & 67,936 & 67,480 & 68,845 & 74,003 & 76,049 \\
\hline $\begin{array}{l}\text { D: supply of electricity, gas, steam and air } \\
\text { conditioning }\end{array}$ & 546 & 559 & 543 & 570 & 567 & 596 & 613 \\
\hline $\begin{array}{l}\text { E: supply of water, sewerage, waste management } \\
\text { and environmental remediation services }\end{array}$ & 2,482 & 2,433 & 2,398 & 2,415 & 2,432 & 2,604 & 2,676 \\
\hline F: construction & 23,703 & 21,254 & 19,455 & 19,017 & 19,347 & 22,007 & 22,616 \\
\hline $\begin{array}{l}\text { G: wholesale and retail trade, repair of motor } \\
\text { vehicles and motorcycles }\end{array}$ & 38,867 & 37,553 & 36,503 & 36,568 & 38,349 & 40,221 & 41,334 \\
\hline H: transport and storage & 12,629 & 12,435 & 12,447 & 12,869 & 13,478 & 13,674 & 14,052 \\
\hline I: accommodation and food service businesses & 19,759 & 19,200 & 19,017 & 19,758 & 21,747 & 21,301 & 21,890 \\
\hline $\mathrm{J}$ : information and communications services & 6,006 & 6,094 & 5,977 & 6,102 & 6,580 & 6,586 & 6,768 \\
\hline K: financial and insurance service businesses & 2,436 & 2,394 & 2,378 & 2,374 & 2,400 & 2,566 & 2,637 \\
\hline L: real estate businesses & 696 & 690 & 642 & 636 & 700 & 720 & 740 \\
\hline M: professional, scientific and technical businesses & 7,964 & 7,922 & 7,741 & 7,914 & 8,566 & 8,588 & 8,825 \\
\hline $\begin{array}{l}\text { N: rental and travel agencies, business support } \\
\text { services }\end{array}$ & 11,318 & 11,280 & 11,177 & 11,201 & 11,632 & 12,121 & 12,456 \\
\hline P: education & 1,651 & 1,720 & 1,737 & 1,785 & 2,001 & 1,904 & 1,957 \\
\hline Q: healthcare and social services & 6,240 & 6,401 & 6,401 & 6,585 & 7,047 & 6,996 & 7,190 \\
\hline $\begin{array}{l}\text { R: arts, sports, entertainment and amusement } \\
\text { businesses }\end{array}$ & 2,396 & 2,322 & 2,168 & 2,011 & 2,168 & 2,369 & 2,435 \\
\hline S: other service businesses & 3,472 & 3,360 & 3,425 & 3,495 & 3,728 & 3,743 & 3,846 \\
\hline TOTAL & 212,722 & 205,432 & 200,427 & 201,254 & 210,041 & 220,523 & 226,621 \\
\hline
\end{tabular}




\section{Figure 8}

\begin{tabular}{|l|l|l|l|l|l|l|l|}
\hline \multirow{2}{*}{$\begin{array}{l}\text { years/number of macro enterprises } \\
\text { in Italy vectors }\end{array}$} & 2012 & 2013 & 2014 & 2015 & 2016 & 2017 & 2018 \\
\cline { 2 - 8 } & 212,722 & 205,432 & 200,427 & 201,254 & 210,041 & 220,523 & 226,621 \\
\hline
\end{tabular}

\section{Italy - number of macro enterprises}

232,000
228,000
224,000
220,000
216,000
212,000
208,000
204,000
200,000
196,000
192,000
188,000
184,000

$\begin{array}{lllllll}2012 & 2013 & 2014 & 2015 & 2016 & 2017\end{array}$

***** Section figures 5 and 6 - Loans to micro enterprises (self-processed data of Bank of Italy - stock values in thousands of euro)

Figure 9

\begin{tabular}{|c|c|c|c|c|c|c|c|}
\hline $31 / 01 / 2012$ & $3,924,505$ & $31 / 10 / 2013$ & $3,596,882$ & $31 / 07 / 2015$ & $3,523,998$ & $30 / 04 / 2017$ & $3,443,130$ \\
\hline $29 / 02 / 2012$ & $3,919,951$ & $30 / 11 / 2013$ & $3,566,020$ & $31 / 08 / 2015$ & $3,505,632$ & $31 / 05 / 2017$ & $3,452,826$ \\
\hline $31 / 03 / 2012$ & $3,865,810$ & $31 / 12 / 2013$ & $3,556,005$ & $30 / 09 / 2015$ & $3,516,844$ & $30 / 06 / 2017$ & $3,399,004$ \\
\hline $30 / 04 / 2012$ & $3,876,134$ & $31 / 01 / 2014$ & $3,644,238$ & $31 / 10 / 2015$ & $3,498,269$ & $31 / 07 / 2017$ & $3,305,219$ \\
\hline $31 / 05 / 2012$ & $3,880,775$ & $28 / 02 / 2014$ & $3,633,203$ & $30 / 11 / 2015$ & $3,536,971$ & $31 / 08 / 2017$ & $3,294,734$ \\
\hline $30 / 06 / 2012$ & $3,853,389$ & $31 / 03 / 2014$ & $3,628,280$ & $31 / 12 / 2015$ & $3,509,561$ & $30 / 09 / 2017$ & $3,291,878$ \\
\hline $31 / 07 / 2012$ & $3,819,758$ & $30 / 04 / 2014$ & $3,613,463$ & $31 / 01 / 2016$ & $3,505,739$ & $31 / 10 / 2017$ & $3,305,199$ \\
\hline $31 / 08 / 2012$ & $3,795,494$ & $31 / 05 / 2014$ & $3,579,649$ & $29 / 02 / 2016$ & $3,518,556$ & $30 / 11 / 2017$ & $3,325,509$ \\
\hline $30 / 09 / 2012$ & $3,778,401$ & $30 / 06 / 2014$ & $3,570,033$ & $31 / 03 / 2016$ & $3,521,410$ & $31 / 12 / 2017$ & $3,305,659$ \\
\hline $31 / 10 / 2012$ & $3,786,101$ & $31 / 07 / 2014$ & $3,548,786$ & $30 / 04 / 2016$ & $3,507,487$ & $31 / 01 / 2018$ & $3,311,735$ \\
\hline $30 / 11 / 2012$ & $3,779,619$ & $31 / 08 / 2014$ & $3,518,787$ & $31 / 05 / 2016$ & $3,518,370$ & $28 / 02 / 2018$ & $3,296,940$ \\
\hline $31 / 12 / 2012$ & $3,722,310$ & $30 / 09 / 2014$ & $3,534,184$ & $30 / 06 / 2016$ & $3,493,093$ & $31 / 03 / 2018$ & $3,304,006$ \\
\hline $31 / 01 / 2013$ & $3,731,535$ & $31 / 10 / 2014$ & $3,527,510$ & $31 / 07 / 2016$ & $3,438,590$ & $30 / 04 / 2018$ & $3,307,596$ \\
\hline $28 / 02 / 2013$ & $3,722,496$ & $30 / 11 / 2014$ & $3,522,209$ & $31 / 08 / 2016$ & $3,435,170$ & $31 / 05 / 2018$ & $3,318,292$ \\
\hline $31 / 03 / 2013$ & $3,705,210$ & $31 / 12 / 2014$ & $3,519,180$ & $30 / 09 / 2016$ & $3,421,025$ & $30 / 06 / 2018$ & $3,029,209$ \\
\hline
\end{tabular}




\begin{tabular}{|l|l|l|l|l|l|l|l}
$\mathbf{3 0 / 0 4 / 2 0 1 3}$ & $3,709,212$ & $\mathbf{3 1 / 0 1 / 2 0 1 5}$ & $3,527,181$ & $31 / 10 / 2016$ & $3,413,568$ & $31 / 07 / 2018$ & $3,016,395$ \\
\hline $\mathbf{3 1 / 0 5 / 2 0 1 3}$ & $3,704,006$ & $\mathbf{2 8 / 0 2 / 2 0 1 5}$ & $3,518,679$ & $\mathbf{3 0 / 1 1 / 2 0 1 6}$ & $3,429,177$ & $\mathbf{3 1 / 0 8 / 2 0 1 8}$ & $3,004,079$ \\
\hline $\mathbf{3 0 / 0 6 / 2 0 1 3}$ & $3,662,903$ & $\mathbf{3 1 / 0 3 / 2 0 1 5}$ & $3,545,684$ & $\mathbf{3 1 / 1 2 / 2 0 1 6}$ & $3,410,455$ & $\mathbf{3 0 / 0 9 / 2 0 1 8}$ & $2,995,745$ \\
\hline $\mathbf{3 1 / 0 7 / 2 0 1 3}$ & $3,634,410$ & $\mathbf{3 0 / 0 4 / 2 0 1 5}$ & $3,544,313$ & $\mathbf{3 1 / 0 1 / 2 0 1 7}$ & $3,431,125$ & $\mathbf{3 1 / 1 0 / 2 0 1 8}$ & $3,018,993$ \\
\hline $\mathbf{3 1 / 0 8 / 2 0 1 3}$ & $3,601,313$ & $\mathbf{3 1 / 0 5 / 2 0 1 5}$ & $3,526,313$ & $\mathbf{2 8 / 0 2 / 2 0 1 7}$ & $3,442,245$ & $\mathbf{3 0 / 1 1 / 2 0 1 8}$ & $3,041,101$ \\
\hline $\mathbf{3 0 / 0 9 / 2 0 1 3}$ & $3,593,634$ & $\mathbf{3 0 / 0 6 / 2 0 1 5}$ & $3,557,350$ & $\mathbf{3 1 / 0 3 / 2 0 1 7}$ & $3,441,119$ & $\mathbf{3 1 / 1 2 / 2 0 1 8}$ & $\mathbf{2 , 8 9 5 , 2 8 6}$ \\
\hline
\end{tabular}

Figure 10

\section{Sardinia - Loans to micro enterprises}

4500000

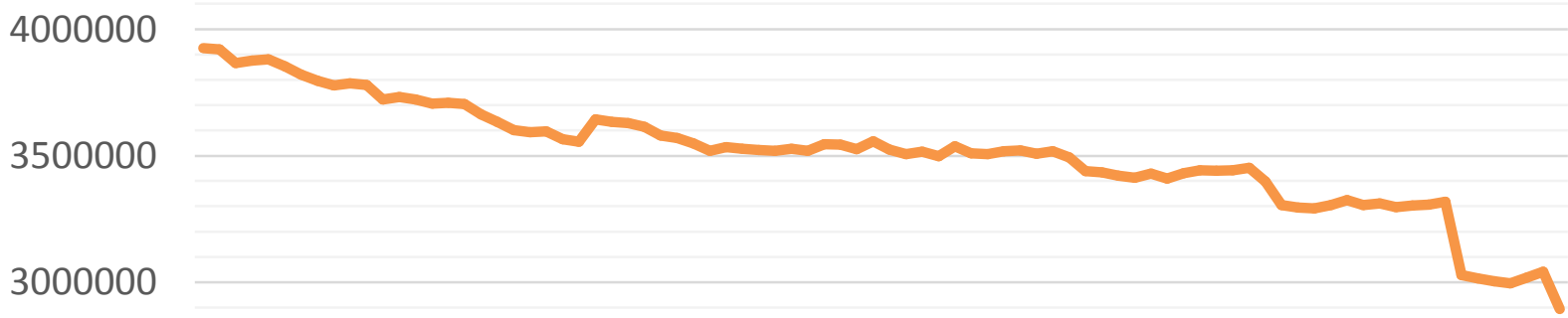

2500000

2000000

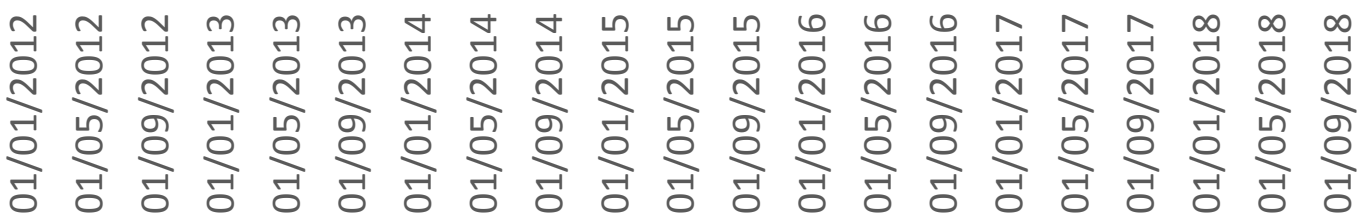

Figure 11

\begin{tabular}{|c|c|c|c|c|c|c|c|}
\hline $1 / 03 / 2012$ & $196,104,283$ & $31 / 12 / 2013$ & $185,086,851$ & $30 / 09 / 2015$ & $177,848,224$ & $30 / 06 / 2017$ & $164,846,165$ \\
\hline 30/06/2012 & $194,108,548$ & $31 / 03 / 2014$ & $186,303,319$ & $31 / 12 / 2015$ & $176,092,382$ & $30 / 09$ & $158,686,183$ \\
\hline /09/20 & $1,839,155$ & $30 / 06 / 2014$ & $183,362,737$ & $31 / 03 / 2016$ & 173,6 & $31 / 12$ & 150 \\
\hline $1 / 12 / 2012$ & $192,277,603$ & $30 / 09 / 2014$ & $181,991,189$ & $30 / 06 / 2016$ & $172,330,425$ & $31 / 0$ & 157,2 \\
\hline $1 / 03 / 2013$ & $, 075,414$ & $31 / 12 / 2014$ & $180,982,518$ & $30 / 09 / 2016$ & $170,408,469$ & $30 / 06$ & $151,569,685$ \\
\hline 0 & 74 & & 18 & 16 & 31 & & \\
\hline & $86,463,427$ & 15 & 410 & & 0,16 & 018 & 44,0 \\
\hline
\end{tabular}




\section{Figure 12}

21500000
19500000
17500000
15500000
13500000
11500000
95000000

\section{Italy - Loans to micro enterprises}

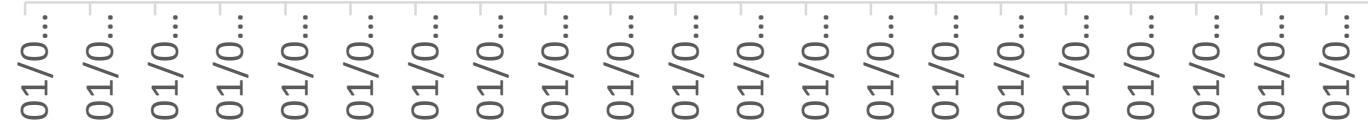

$* * * * *$

Section figures 7 and 8 - Loans to macro enterprises (self-processed data of Bank of Italy-stock values in thousands of euro)

Figure 13

Sardinia: monthly surveys of loans to macro enterprises - stock values in thousands of euro

\begin{tabular}{|c|c|c|c|c|c|c|c|}
\hline $31 / 01 / 2012$ & $20,945,376$ & $31 / 10 / 2013$ & $18,626,870$ & $31 / 07 / 2015$ & $19,223,444$ & $30 / 04 / 2017$ & $18,641,376$ \\
\hline $29 / 02 / 2012$ & $20,782,407$ & $30 / 11 / 2013$ & $18,294,656$ & $31 / 08 / 2015$ & $19,175,133$ & $31 / 05 / 2017$ & $18,783,067$ \\
\hline $31 / 03 / 2012$ & $19,755,188$ & $31 / 12 / 2013$ & $18,043,543$ & $30 / 09 / 2015$ & $19,032,800$ & $30 / 06 / 2017$ & $17,918,440$ \\
\hline $30 / 04 / 2012$ & $20,148,404$ & $31 / 01 / 2014$ & $19,254,395$ & $31 / 10 / 2015$ & $18,949,734$ & $31 / 07 / 2017$ & $17,910,313$ \\
\hline $31 / 05 / 2012$ & $19,926,114$ & $28 / 02 / 2014$ & $19,358,613$ & $30 / 11 / 2015$ & $19,003,366$ & $31 / 08 / 2017$ & $17,738,670$ \\
\hline $30 / 06 / 2012$ & $19,240,685$ & $31 / 03 / 2014$ & $18,982,302$ & $31 / 12 / 2015$ & $19,099,174$ & $30 / 09 / 2017$ & $17,243,961$ \\
\hline $31 / 07 / 2012$ & $19,482,460$ & $30 / 04 / 2014$ & $19,752,931$ & $31 / 01 / 2016$ & $19,112,655$ & $31 / 10 / 2017$ & $17,560,839$ \\
\hline $31 / 08 / 2012$ & $19,184,105$ & $31 / 05 / 2014$ & $19,601,713$ & $29 / 02 / 2016$ & $19,015,982$ & $30 / 11 / 2017$ & $17,261,327$ \\
\hline $30 / 09 / 2012$ & $19,126,712$ & $30 / 06 / 2014$ & $18,825,923$ & $31 / 03 / 2016$ & $19,080,784$ & $31 / 12 / 2017$ & $16,993,650$ \\
\hline $31 / 10 / 2012$ & $19,253,365$ & $31 / 07 / 2014$ & $18,969,477$ & $30 / 04 / 2016$ & $19,130,911$ & $31 / 01 / 2018$ & $16,970,089$ \\
\hline $30 / 11 / 2012$ & $19,423,965$ & $31 / 08 / 2014$ & $19,579,825$ & $31 / 05 / 2016$ & $19,202,102$ & $28 / 02 / 2018$ & $17,048,694$ \\
\hline $31 / 12 / 2012$ & $18,902,468$ & $30 / 09 / 2014$ & $18,596,942$ & $30 / 06 / 2016$ & $19,226,436$ & $31 / 03 / 2018$ & $16,844,715$ \\
\hline $31 / 01 / 2013$ & $19,268,629$ & $31 / 10 / 2014$ & $19,050,007$ & $31 / 07 / 2016$ & $18,919,625$ & $30 / 04 / 2018$ & $17,179,620$ \\
\hline $28 / 02 / 2013$ & $19,238,602$ & $30 / 11 / 2014$ & $18,619,485$ & $31 / 08 / 2016$ & $18,724,079$ & $31 / 05 / 2018$ & $17,632,867$ \\
\hline $31 / 03 / 2013$ & $18,896,487$ & 2014 & $18,416,271$ & 2016 & 18 & 2018 & $16,543,379$ \\
\hline $30 / 04 / 2013$ & $19,209,658$ & $31 / 01 / 2015$ & $18,800,787$ & $31 / 10 / 2016$ & $18,805,788$ & $31 / 07 / 2018$ & $15,872,481$ \\
\hline $1 / 05 / 2013$ & $18,891,729$ & $28 / 02 / 2015$ & $18,647,498$ & $30 / 11 / 2016$ & $18,629,860$ & $31 / 08 / 2018$ & $15,763,331$ \\
\hline
\end{tabular}




\begin{tabular}{|c|c|c|c|c|c|c|c|}
\hline $30 / 06 / 2013$ & $18,818,546$ & $31 / 03 / 2015$ & $18,962,928$ & $31 / 12 / 2016$ & $18,339,360$ & $30 / 09 / 2018$ & $14,822,036$ \\
\hline $31 / 07 / 2013$ & $18,726,947$ & $30 / 04 / 2015$ & $18,963,265$ & $31 / 01 / 2017$ & $18,799,061$ & $31 / 10 / 2018$ & $15,383,655$ \\
\hline $31 / 08 / 2013$ & $18,832,875$ & $31 / 05 / 2015$ & $18,900,447$ & $28 / 02 / 2017$ & $18,727,914$ & $30 / 11 / 2018$ & $15,390,497$ \\
\hline $30 / 09 / 2013$ & $18,288,769$ & $30 / 06 / 2015$ & $19,195,611$ & $31 / 03 / 2017$ & $17,952,327$ & $31 / 12 / 2018$ & $14,189,483$ \\
\hline
\end{tabular}

Figure 14

\section{Sardinia - Loans to macro enterprises}

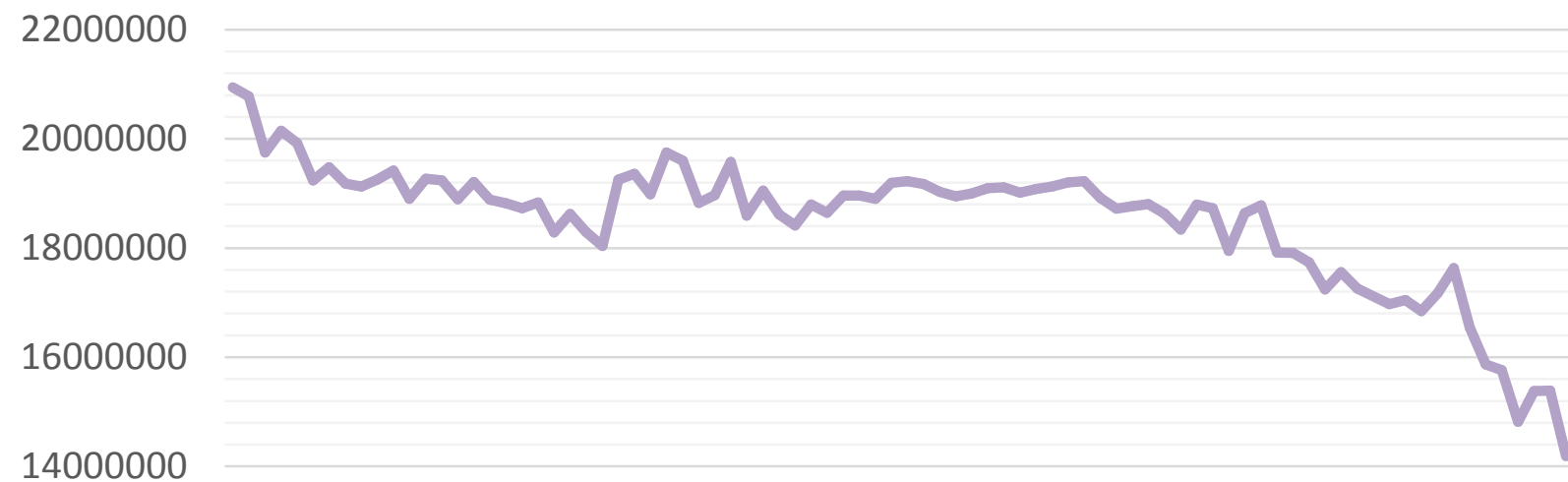

12000000

10000000

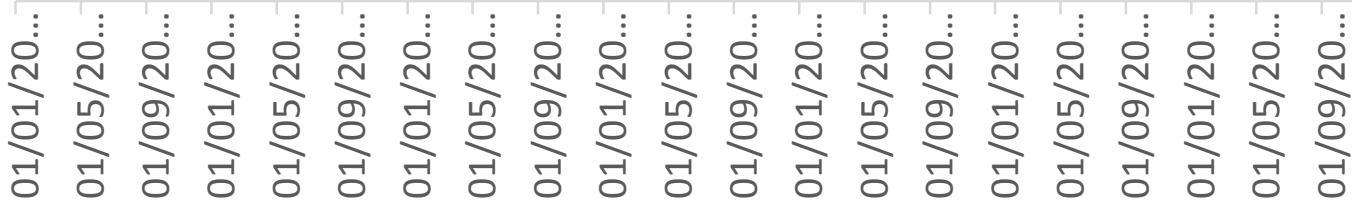

Figure 15

Italy: quarterly surveys of loans to macro enterprises - stock values in thousands of euro

\begin{tabular}{|c|c|c|c|c|c|c|c|}
\hline$/ 0$ &, 366 & $1 / 12 / 2013$ & $1,521,713,568$ & $30 / 09 / 2015$ & $1,501,913,382$ & $30 / 06 / 2017$ & 8,448 \\
\hline $30 / 06 / 2012$ & $645,185,055$ & $n z$ & $1,553,078,446$ & $31 / 12 / 2015$ & 1,483 & 017 & 4,098 \\
\hline $30 / 09 / 2012$ & 82 & 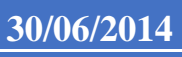 & 6 & 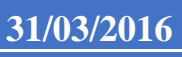 & 67 & $31 / 12 / 2017$ & 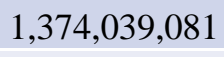 \\
\hline $31 / 12$ & $1,611,501,427$ & 30 & 1,541 & $30 / 0$ & $1,4 \varepsilon$ & 10 & 809 \\
\hline $31 /$ & 8 & ר & 509,099, & $30 / 09 / 2$ & $465,279,100$ & r & $1,316,758$ \\
\hline $30 / 0$ & 1,5 & 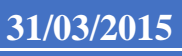 & 1,5 & 31 & 1,4 & & 1,30 \\
\hline 13 & $1,550,624,325$ & 201 & $1,514,910,90$ & $31 / 03 / 2017$ & 7,652 & $31 /$ & $1,268,272,037$ \\
\hline
\end{tabular}


Figure 16

\section{Italy - Loans to macro enterprises}
$1.8 \mathrm{E}+09$
$1.7 \mathrm{E}+09$
$1.6 \mathrm{E}+09$
$1.5 \mathrm{E}+09$
$1.4 \mathrm{E}+09$
$1.3 \mathrm{E}+09$
$1.2 \mathrm{E}+09$
$1.1 \mathrm{E}+09$
$1 \mathrm{E}+09$

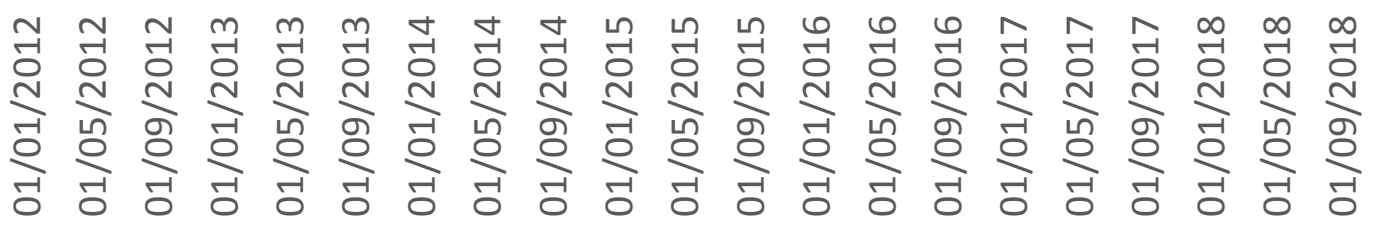

$* * * * *$

Section figures 9 and 10 - Net non-performing loans to micro enterprises (self-processed data of Bank of Italy - stock values in thousands of euro)

Figure 17

\begin{tabular}{|l|l|l|l|l|l|}
\hline \multicolumn{6}{|c|}{ Sardinia: net non performing loans to micro enterprises - in thousands of euro } \\
\hline $31 / 03 / 2012$ & 415 & $31 / 12 / 2013$ & 424 & $30 / 09 / 2015$ & 488 \\
\hline $30 / 06 / 2012$ & 421 & $31 / 03 / 2014$ & 434 & $31 / 12 / 2015$ & 548 \\
\hline $30 / 09 / 2012$ & 429 & $30 / 06 / 2014$ & 436 & $31 / 03 / 2016$ & 557 \\
\hline $31 / 12 / 2012$ & 442 & $30 / 09 / 2014$ & 443 & $30 / 06 / 2016$ & 551 \\
\hline $31 / 03 / 2013$ & 405 & $31 / 12 / 2014$ & 453 & $30 / 09 / 2016$ & 523 \\
\hline $30 / 06 / 2013$ & 412 & $31 / 03 / 2015$ & 464 & $31 / 12 / 2016$ & 524 \\
\hline $30 / 09 / 2013$ & 408 & $30 / 06 / 2015$ & 479 & $31 / 03 / 2017$ & 514
\end{tabular}

Figure 18

600

550

500

450

400

350

300

\section{Sardinia - non performing loans micro enterprises}

ว 
Figure 19

\begin{tabular}{|l|l|l|l|l|l|}
\hline \multicolumn{7}{|l}{ Italy: net non performing loans to micro enterprises - in thousands of euro } \\
\hline $\mathbf{3 1 / 0 3 / 2 0 1 2}$ & 10,473 & $\mathbf{3 1 / 1 2 / 2 0 1 3}$ & 13,253 & $\mathbf{3 0 / 0 9 / 2 0 1 5}$ & 14,774 \\
\hline $\mathbf{3 0 / 0 6 / 2 0 1 2}$ & 10,825 & $\mathbf{3 1 / 0 3 / 2 0 1 4}$ & 13,624 & $\mathbf{3 1 / 1 2 / 2 0 1 5}$ & 14,859 \\
\hline $\mathbf{3 0 / 0 9 / 2 0 1 2}$ & 11,126 & $\mathbf{3 0 / 0 6 / 2 0 1 4}$ & 13,869 & $\mathbf{3 1 / 0 3 / 2 0 1 6}$ & 14,683 \\
\hline $\mathbf{3 1 / 1 2 / 2 0 1 2}$ & 11,743 & $\mathbf{3 0 / 0 9 / 2 0 1 4}$ & 14,214 & $\mathbf{3 0 / 0 6 / 2 0 1 6}$ & 14,603 \\
\hline $\mathbf{3 1 / 0 3 / 2 0 1 3}$ & 11,967 & $\mathbf{3 1 / 1 2 / 2 0 1 4}$ & 13,680 & $\mathbf{3 0 / 0 9 / 2 0 1 6}$ & 14,754 \\
\hline $\mathbf{3 0 / 0 6 / 2 0 1 3}$ & 12,350 & $\mathbf{3 1 / 0 3 / 2 0 1 5}$ & 14,076 & $\mathbf{3 1 / 1 2 / 2 0 1 6}$ & 15,229 \\
\hline $\mathbf{3 0 / 0 9 / 2 0 1 3}$ & 12,692 & $\mathbf{3 0 / 0 6 / 2 0 1 5}$ & 14,425 & $\mathbf{3 1 / 0 3 / 2 0 1 7}$ & 14,845 \\
\hline
\end{tabular}

Figure 20

\section{Italy - non performing loans micro enterprises}

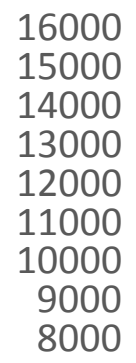

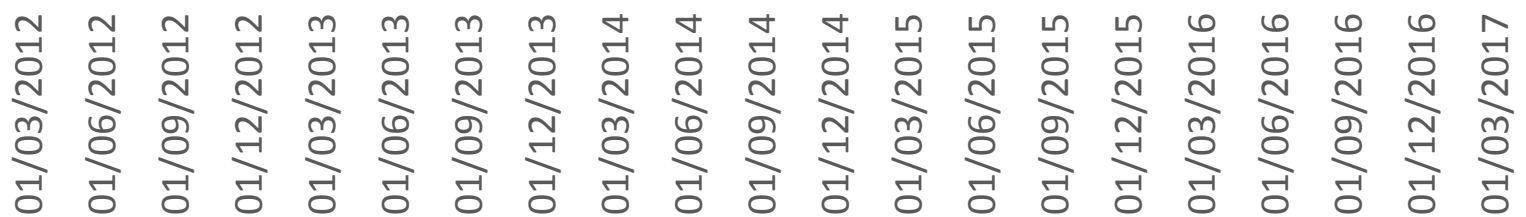

*****Section figures 11 and 12 - Net non-performing loans to macro enterprises (self-processed data of Bank of Italy stock values in thousands of euro)

'Figure 21

Sardinia: net non performing loans to macro enterprises - in thousands of euro

\begin{tabular}{|l|l|l|l|l|l|}
\hline $\mathbf{3 1 / 0 3 / 2 0 1 2}$ & 1,455 & $\mathbf{3 1 / 1 2 / 2 0 1 3}$ & 1,609 & $30 / 09 / 2015$ & 2,209 \\
\hline $\mathbf{3 0 / 0 6 / 2 0 1 2}$ & 1,549 & $\mathbf{3 1 / 0 3 / 2 0 1 4}$ & 1,681 & $31 / 12 / 2015$ & 2,814 \\
\hline $\mathbf{3 0 / 0 9 / 2 0 1 2}$ & 1,627 & $\mathbf{3 0 / 0 6 / 2 0 1 4}$ & 1,774 & $\mathbf{3 1 / 0 3 / 2 0 1 6}$ & 2,834 \\
\hline $\mathbf{3 1 / 1 2 / 2 0 1 2}$ & 1,618 & $\mathbf{3 0 / 0 9 / 2 0 1 4}$ & 1,908 & $\mathbf{3 0 / 0 6 / 2 0 1 6}$ & 2,832 \\
\hline $\mathbf{3 1 / 0 3 / 2 0 1 3}$ & 1,334 & $\mathbf{3 1 / 1 2 / 2 0 1 4}$ & 2,057 & $\mathbf{3 0 / 0 9 / 2 0 1 6}$ & 2,815 \\
\hline $\mathbf{3 0 / 0 6 / 2 0 1 3}$ & 1,405 & $\mathbf{3 1 / 0 3 / 2 0 1 5}$ & 2,045 & $\mathbf{3 1 / 1 2 / 2 0 1 6}$ & 2,952 \\
\hline $\mathbf{3 0 / 0 9 / 2 0 1 3}$ & 1,499 & $\mathbf{3 0 / 0 6 / 2 0 1 5}$ & 2,137 & $31 / 03 / 2017$ & 2,938 \\
\hline
\end{tabular}

Figure 22 


\section{Sardinia - non performing loans macro enterprises}

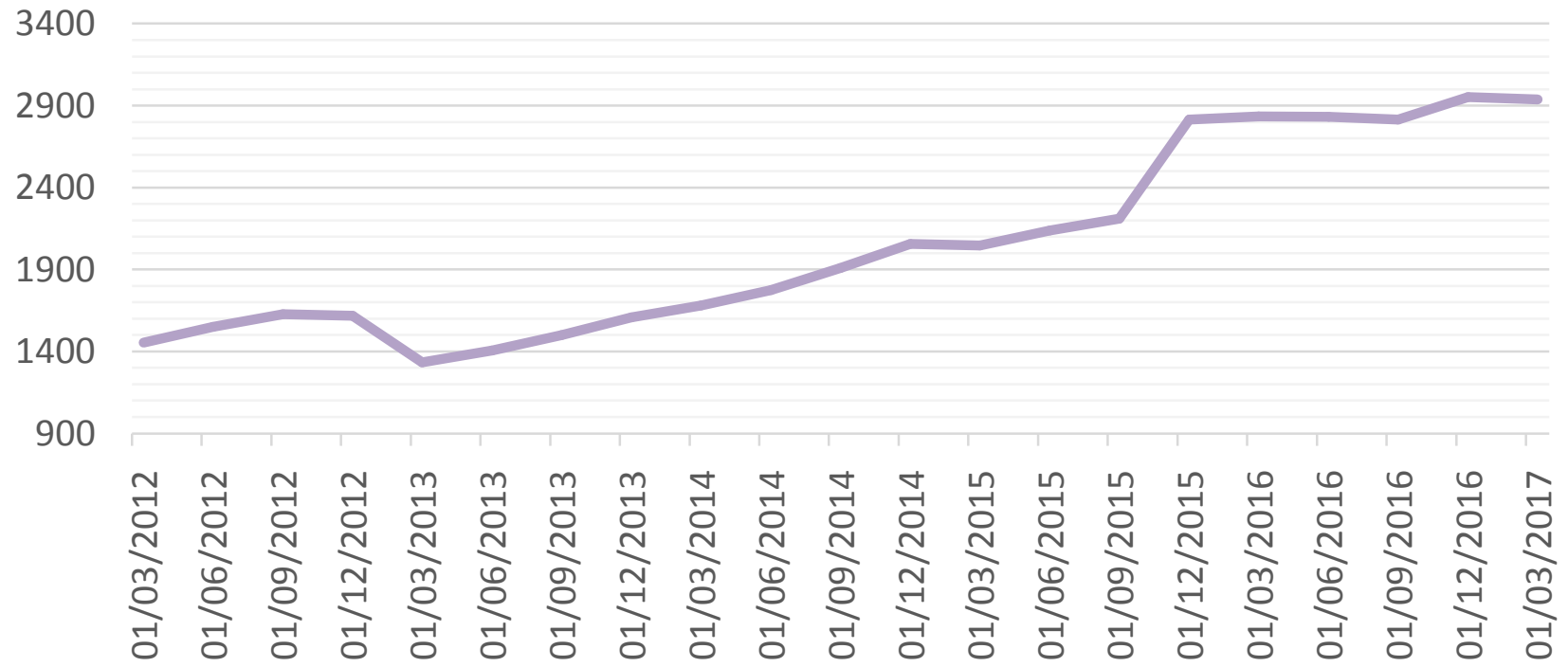

Figure 23

\begin{tabular}{|l|l|l|l|l|l|}
\hline \multicolumn{7}{l}{ Italy: net non performing loans macro enterprises - in thousands of euro } \\
\hline $\mathbf{3 1 / 0 3 / 2 0 1 2}$ & 69,899 & $\mathbf{3 1 / 1 2 / 2 0 1 3}$ & 104,258 & $\mathbf{3 0 / 0 9 / 2 0 1 5}$ & 134,512 \\
\hline $\mathbf{3 0 / 0 6 / 2 0 1 2}$ & 74,349 & $\mathbf{3 1 / 0 3 / 2 0 1 4}$ & 111,726 & $\mathbf{3 1 / 1 2 / 2 0 1 5}$ & $\mathbf{1 3 6 , 5 6 4}$ \\
\hline $\mathbf{3 0 / 0 9 / 2 0 1 2}$ & 77,502 & $\mathbf{3 0 / 0 6 / 2 0 1 4}$ & 116,406 & $\mathbf{3 1 / 0 3 / 2 0 1 6}$ & 133,185 \\
\hline $\mathbf{3 1 / 1 2 / 2 0 1 2}$ & 81,677 & $\mathbf{3 0 / 0 9 / 2 0 1 4}$ & 119,310 & $\mathbf{3 0 / 0 6 / 2 0 1 6}$ & 135,080 \\
\hline $\mathbf{3 1 / 0 3 / 2 0 1 3}$ & 85,363 & $\mathbf{3 1 / 1 2 / 2 0 1 4}$ & 122,643 & $\mathbf{3 0 / 0 9 / 2 0 1 6}$ & 136,487 \\
\hline $\mathbf{3 0 / 0 6 / 2 0 1 3}$ & 91,292 & $\mathbf{3 1 / 0 3 / 2 0 1 5}$ & 126,021 & $\mathbf{3 1 / 1 2 / 2 0 1 6}$ & 138,805 \\
\hline $\mathbf{3 0 / 0 9 / 2 0 1 3}$ & 96,203 & $\mathbf{3 0 / 0 6 / 2 0 1 5}$ & 131,237 & $\mathbf{3 1 / 0 3 / 2 0 1 7}$ & 135,640 \\
\hline
\end{tabular}

Figure 24

\section{Italy - non performing loans macro enterprises}

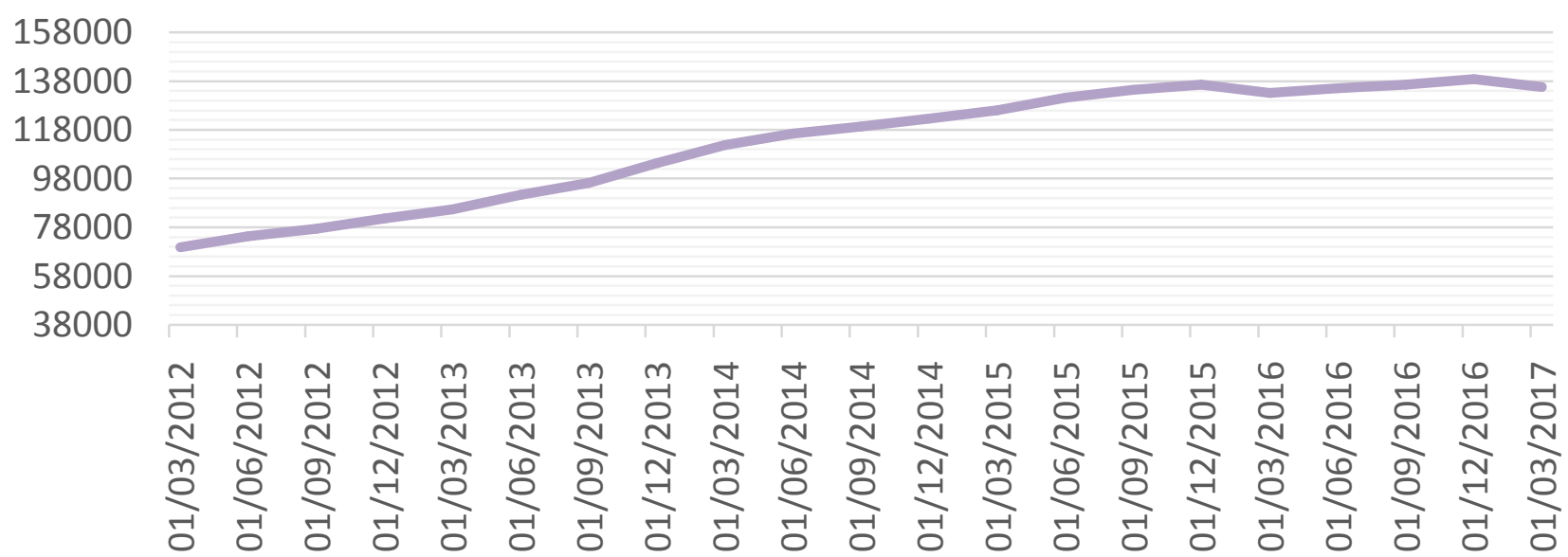


The scenario presented above, begins from the progressive reduction in the disbursement of loans - which we assume to be an independent variable - to which a generalised increase in net non-performing loans can be reasonably correlated ${ }^{2}$ (average Bravais-Pearson correlation coefficient $\mathrm{C}_{\mathrm{BP}}=-0.57$ ). Nevertheless, the repercussion of this manifestation had different results on the size of the productive sector. On the one hand, micro enterprises tend to leave the market more quickly than new-companies of a similar size. At the same time, macro enterprises seem to not be affected by this phenomenon. Based on this observation, a theoretical model that explains the basis of these findings was developed.

From the data-set adopted, the correlation index between the reduction in the volumes of credit disbursed to the production system and the number of enterprises operating on the market in Italy was: +0.73 for micro enterprises and 0.71 for macro enterprises ${ }^{3}$.

It should be taken into account that more than $90 \%$ of the makeup of Italian and Sardinian production comprises micro enterprises. In the overall statistical data, the granularity of even the largest enterprises is insufficient to compensate and support the general system, as the figures below show clearly.

Section figures 13 and 14 - Total number of enterprises (normalization from ISTAT and Chambers of Commerce sources)

Figure 25

\begin{tabular}{|l|l|l|l|l|l|l|l|}
\hline & \multicolumn{7}{|c|}{ years/number of enterprises in Sardinia vectors } \\
\hline & $\mathbf{2 0 1 2}$ & $\mathbf{2 0 1 3}$ & $\mathbf{2 0 1 4}$ & $\mathbf{2 0 1 5}$ & $\mathbf{2 0 1 6}$ & $\mathbf{2 0 1 7}$ & $\mathbf{2 0 1 8}$ \\
\hline $\begin{array}{l}\text { Micro } \\
\text { enterprises }\end{array}$ & 102,847 & 101,647 & 99,404 & 98,701 & 100,305 & 100,045 & 100,169 \\
\hline $\begin{array}{l}\text { Macro } \\
\text { enterprises }\end{array}$ & 3,751 & 3,557 & 3,370 & 3,316 & 3,497 & 3,746 & 3,784 \\
\hline $\begin{array}{l}\text { Total } \\
\text { enterprises }\end{array}$ & 106,598 & 105,204 & 102,774 & 102,017 & 103,802 & 103,791 & 103,953 \\
\hline
\end{tabular}

\section{Sardinia - total number of enterprises}

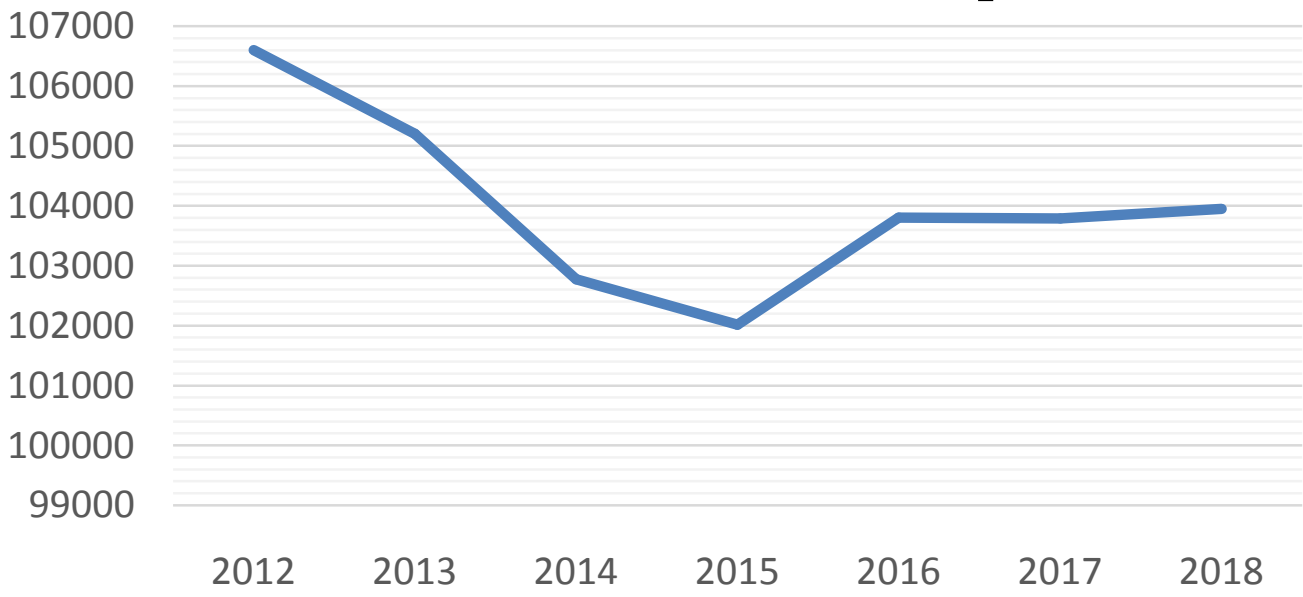

Figure 26

\begin{tabular}{|l|l|l|l|l|l|l|l|}
\hline & \multicolumn{7}{|c|}{ years/number of enterprises in Italy vectors } \\
\hline & 2012 & 2013 & 2014 & 2015 & 2016 & 2017 & 2018 \\
\hline $\begin{array}{l}\text { Micro } \\
\text { enterprises }\end{array}$ & 212,722 & 205,432 & 200,427 & 201,254 & 210,041 & 220,523 & 226,621 \\
\hline $\begin{array}{l}\text { Macro } \\
\text { enterprises }\end{array}$ & $4,229,730$ & $4,185,081$ & $4,158,660$ & $4,136,831$ & $4,142,556$ & $4,141,465$ & $4,140,633$ \\
\hline $\begin{array}{l}\text { Total } \\
\text { enterprises }\end{array}$ & $4,442,452$ & $4,390,513$ & $4,359,087$ & $4,338,085$ & $4,352,597$ & $4,361,988$ & $4,367,254$ \\
\hline
\end{tabular}




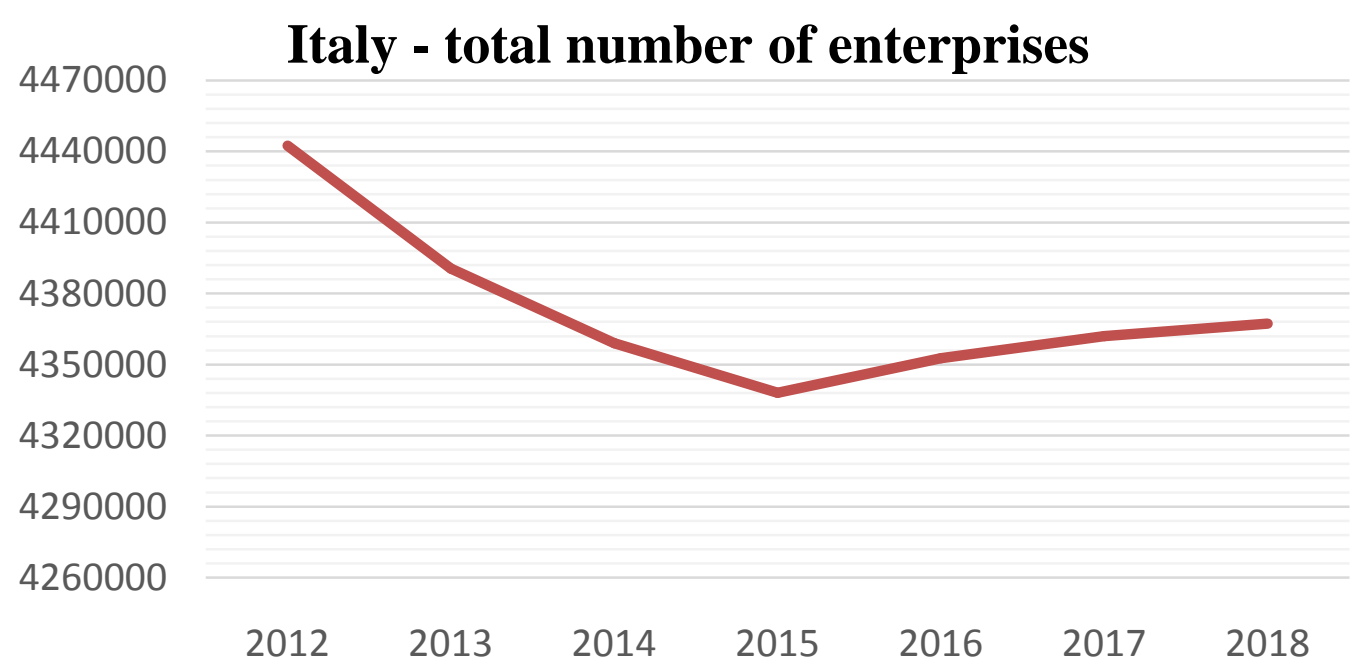

Therefore, for all the reasons detailed above, stability, or rather growth, in a real economy founded on micro and very small enterprises, is a function - though not univariate - of the average credit disbursed. Less available credit significant impacts the real economy, especially, in countries where the financial system is centred on banks.Bond and equity markets are underdeveloped and therefore cannot provide alternative resources, especially for small businesses ${ }^{4}$. The worsening of the conditions of access to credit, for indebted and vulnerable subjects, as could be the micro enterprises, can leads significant effects: as the reduction in consumption and investments in the real economy. The Internal Rating-based ( IRB ) "basic" or "advanced", applied by banks, to their customers, it is made without distinctions due to the size of the operator requesting financial support. The assigned rating class determines whether a costumer can access credit risk and how much it will cost. The assigned rating class determines whether anenterprise can access credit and how much it will cost. This therefore orients the performance results for economic micro-entities, structurally unable to replace financial resources with endogenous means, in the same way as the Probability of Default (PD) assigned by the algorithm, thus reversing the cause and effect principle. In the next section we formalize this statement from a mathematical point of view.

\section{The model}

Banks implement their strategies for making loans based on commercial and profit-based rationales. Loans are represented by risk portfolios whose overall weighting is derived from the sum of segmented portions, which in turn are based on precise evaluations of each individual counterparty. In the model below, we will proceed by approximating the portfolio average and imagining that all the $n$ positions comprising it $^{5}$ have the same $\mathrm{PD}$, the same Loss Given Default $(L G D)$ and the same, Exposure at Default $(E A D)$, the total expected losses:TE $L_{n}$ will be6:

$$
T E L_{n}=n * P D * E A D * L G D(1)
$$

Because these are expected losses, they represent a cost and should be recorded in the bank's Income Statement. A separate rationale should be applied to unexpected losses, which represent a capital constraint for banks, and which determine the caution that they more frequently adopt when making loans.

At this point, we have chosen here to apply the "Vasicek model"7, about which we are offering an integrative emanation, which describes the different mathematical effects of credit intermediation, with the subsequent disaggregation of the peculiarities of the micro and very small enterprises from the total ${ }^{8}$. Our portfolio comprises the $n$ positions (financed enterprises) described above and the observation we conduct is over a time $t=1$ year:

$$
T L_{n}=\sum_{i=1}^{n} U_{i} L G D_{i} E A D_{i}(2)
$$

Where:

$T L_{n}$ is the total loss on the portfolio over the time $t ; L G D_{i}$ and $E A D_{i}$ are the LGD and the EAD of the i-th company respectively; $U_{i}$ is the Boolean indicator, which takes on a value of 1 if the $\mathrm{i}$-th enterprise has reached default within time $t$, or the value 0 if the $\mathrm{i}$-th enterprise has continued to be performing in the range considered. 
Now, let us suppose that the default is essentially determined, for all the companies in the portfolio, by the size of the budget surplus ( $S_{i}$ for the i-th enterprise): below a certain size, the business does not hold up and goes into default. $S_{i}$ represents a random variable, making it necessary to proceed with the description of its evolutionary process. Note that this is a Markovian process ${ }^{9}$ : at the instant $t_{0}$ the future random value of $S_{i}$ in $t_{1}$ depends only on the state of $S_{i}$ in $t_{0}$ and not on what occurred previously.

Now, let us introduce the amount $X$ - to which we assign great significance for the progress of our thesis - which represents a variable of macroeconomic context and which indicates the further scenario in which the enterprises in the portfolio operate. From this there will be derived an additional random element $Y$, whose variation $\Delta Y$ in an interval $\Delta t$ is the result of the sum of the variations of the elementary states of $Y$ (independent among themselves) and whose variance is analogously the sum of the elementary variances: if $\Delta Y(\delta t)$ has a variance $\sigma^{2}$, over the entire interval $\Delta T$, we will see a total variance of $\Delta Y$ equal to $\sigma^{2} \Delta t$ and, with the same probability distribution of the elementary variations and a corresponding standard deviation of $\sigma \sqrt{\Delta t}$. Therefore:

$$
d Y=X \sigma \sqrt{\Delta t}(3)
$$

With $X$ being a random Gaussian variable.

The overall process is described by the stochastic differential equation:

$$
d S_{i}=S_{i}(t) r_{i} d t+S_{i}(t) X_{i} \sigma_{i} \sqrt{d t}^{10}(4)
$$

Supplementing with Ito's lemma ${ }^{11}$, we find:

$$
S_{i}(T)=S(0) e^{r_{i} T-\frac{1}{2} \sigma_{i}^{2} T+\sigma_{i} \sqrt{T} X_{i}}(5)
$$

It emerges that the randomness of the process $S_{i}(T)$ is determined solely by $X_{i}$. By analysing $X_{i}$, we can break it down into:

$$
X_{i}=\alpha K+\beta e_{i}(6)
$$

where $K$ is the random variable.It comes from the macroeconomic context, while the $e_{i}$ element indicates the random idiosyncratic factor of the individual counterparty. It should be mentioned that a certain component of $e_{i}$ is however dependent on $K$.

Assuming that both of these are normal Gaussian variables (with the average $=0$ and the variance $=1$ ) that are independent among themselves, then:

and

$$
\alpha^{2}+\beta^{2}=1
$$

$$
X_{i}=\alpha K+\sqrt{1-\alpha^{2}} e_{i}(8)
$$

With $\alpha$ known a priori, the above formula describes the process that leads to the default: that is, for $X_{i}$ being less than a minimum $M_{i}$

hence,

$$
P D_{i}=\operatorname{Pr}\left(X_{i}<M_{i}\right)(9)
$$

and

$$
P D_{i}=F\left(M_{i}\right)(10)
$$

$$
M_{i}=F^{-1}\left(P D_{i}\right)(11)
$$

with $F$ being the cumulative probability function of the normal Gaussian distribution.

\section{The Deterministic Approach}

Then, based on the above, a default state will be verified when:

$$
\alpha K+\sqrt{1-\alpha^{2}} e_{i}<F^{-1}\left(P D_{i}\right)(12)
$$

The $e_{i}$ factor is peculiar and intrinsic to each enterprise, we can useitfor isolate micro enterprises with respect to medium-large enterprises. In fact, this factor can be more easily defined on the basis of deterministic considerations, which can be made on the general and accounting structures of those same medium-large enterprises, since they are obviously less dependent on exogenous components of their resources and therefore less sensitive to any turbulence in the economic situation and system.

Instead, for micro and very small enterprises, we assumethat the state of $e$ is described by a wave function $\psi(r, t)$, which indicates the amplitude of probability to find the state, at time $t$, of the enterprise $i$ in $r$. Where " $r$ " is a generic point of the $[\kappa, \lambda]$ interval, $\kappa=0$ is the performance state and $\lambda=1$, which represents default. Even though the $r$ state is also "deterministic", in this case, the result of the measurement becomes probabilistic.

$$
P(r, t) \propto|\psi(r, t)|^{2}(13)
$$


Explaining further, for simplicity's sake, let us consider a generic state $|\psi\rangle^{12}$ expressed by the linear combination of the two limit states $|\kappa\rangle$ and $|\lambda\rangle$ (Eigenstates):

$$
|\psi\rangle=\frac{1}{\sqrt{2}}\{|\kappa\rangle+|\lambda\rangle\}(14)
$$

The measuring instrument is the algorithm used by a generic bank Bin order to calculate the rating, where irepresentsthe micro-enterprise requesting a loan. The idiosyncratic component $e_{i}$ of the micro and very small enterprises, can be evaluated at any time, in all points of the interval $[\kappa, \lambda]$. The measurement of a "macroscopic" rating may result in three positions:

- (0) indicated by the $\left|B_{0}\right\rangle$ state, if the instrument reads that the micro-enterprise's state is $|\kappa\rangle$;

- (1) indicated by the $\left|B_{1}\right\rangle$ state, if the instrument reads that the micro-enterprise's state is $|\lambda\rangle$;

$-(0.5)$ characterizes the adjustment of the instrument before it is measured.

The interaction between the phenomenon and the measuring produces:

$$
\begin{gathered}
|\psi(0)\rangle_{1}=|\kappa\rangle\left|B_{0,5}\right\rangle \Rightarrow|\psi(t)\rangle_{1}=|\kappa\rangle\left|B_{0}\right\rangle(15) \\
|\psi(0)\rangle_{2}=|\lambda\rangle\left|B_{0,5}\right\rangle \Rightarrow|\psi(t)\rangle_{2}=|\lambda\rangle\left|B_{1}\right\rangle(16)
\end{gathered}
$$

For the hypothesized linearity, we would have a generic initial state of $|\psi(0)\rangle$, at time $t=0$, resulting from the overlapping of $|\psi(0)\rangle_{1}$ and $|\psi(0)\rangle_{2}$ :

$$
|\psi(0)\rangle=\frac{1}{\sqrt{2}}\left\{|\kappa\rangle\left|B_{0,5}\right\rangle+|\lambda\rangle\left|B_{0,5}\right\rangle\right\}(17)
$$

which evolves, following the measurement done at a time $t>0$, in the state:

$$
|\psi(t)\rangle=\frac{1}{\sqrt{2}}\left\{|\kappa\rangle\left|B_{0}\right\rangle+|\lambda\rangle\left|B_{1}\right\rangle\right\}(18)
$$

But this is not true, since it is not possible to verify a contextual overlap of the (macroscopic) measures (simultaneously is marked $\left|B_{0}\right\rangle$ and $\left.\left|B_{1}\right\rangle\right)$. We conclude that, following the measurement of the rating, the state of the micro enterprise will no longer an overlapping of Eigenstates of the measured quantity, but will "collapse" in $\kappa$ or in $\lambda$ (or, more correctly, at any point $r$ of the interval $[\kappa, \lambda]$ ) according to whether the instrument measures 0 or 1 (or an intermediate quantity, to which $r$, will correspond, of the expected degree of performance) ${ }^{13}$.

Now, we consider again (12):

$$
\alpha K+\sqrt{1-\alpha^{2}} e_{i}<F^{-1}\left(P D_{i}\right)
$$

and formula (2):

$$
T L_{n}=\sum_{i=1}^{n} U_{i} L G D_{i} E A D_{i}
$$

We define $U_{i}$ :

$$
U_{i}=\left\{\begin{array}{c}
1 \quad \text { if } \alpha K+\sqrt{1-\alpha^{2}} e_{i}<F^{-1}\left(P D_{i}\right) \\
0 \text { otherwise }
\end{array}\right.
$$

and we have

$$
T L_{n}=\sum_{i=1}^{n} U_{i}\left(K, e_{i}\right) L G D_{i} E A D_{i}(20)
$$

In order to maximize $e_{i}$ and $K^{14}$, according to the hypothesis that $P D_{i}, L G D_{i}$ and $E A D_{i}$ are known a priori, we consider the economic system as the cohabitation of entities: the banks that need to minimize losses and reduce capital absorption, and the enterprises that need to obtain credit and doing business sustainably and profitably. We remark that $K$, include monetary policy, international economic influences, credibility and trust of the country, etc.

For these reasons we consider a dynamic system in which the bank-enterprise system (and, in turn, families, since we cannot overlook the positive influence, including macroeconomic effects, generated by an efficient banking sector in the transmission of liquidity to the productive complex so that it can remain healthy and performing (and, on the contrasting condition, where there are the negative effects produced by the disappearance of this virtuous cycle) is against micro enterprises (portfolio). 
We denote the population of banks with $Z_{1}$ and the population of micro enterprises (portfolio) $Z_{2}$.

Now, we consider two possible banks strategies:

a) continuing to manage loans using generalist algorithms;

b) reactivating qualitative forms of relationships, trust and credit cooperation.

As exposed by the empirical calculations shown in the section above,in the first case, the result seems to be on the saving on allocations. However, the greater perceived solidity would only be apparent and in the short-term. Both $e$ components - with reference to micro and very small enterprises - and $K$ are affected, negatively, by the a) strategy. A correlation between the reduction of the productive systemcredit and the higher mortality of the micro enterprises (default of the $Z_{2}$ population) and the increase of the non-performing bank loans (default of the $Z_{1}$ population) has been observed. Let us introduce the formal statements that support recourse to the b) strategy: whether $z_{1}=z_{1}(t)$ and $z_{2}=z_{2}(t)$ are manifestations of performing loans ${ }^{15}$ at time $t$ of the two populations $Z_{1}$ and $Z_{2}$ respectively:

$$
\left\{\begin{array}{l}
\frac{d z_{1}}{d t}=z_{1} f_{1}\left(z_{1}, z_{2}\right) \\
\frac{d z_{2}}{d t}=z_{2} f_{2}\left(z_{1}, z_{2}\right)
\end{array}\right.
$$

This system of differential equations ${ }^{16}$ indicates the dependence of the number of performing loans of each population on that of both; the link between the two populations is mutual.

$$
\frac{\partial f_{1}}{\partial z_{2}}>0 \quad \text { and } \quad \frac{\partial f_{2}}{\partial z_{1}}>0(22)
$$

A growth in the performance of $Z_{2}$ corresponds to a growth in $Z_{1}$ (therefore, it is systemic). The mathematical zeros of the model are,

- $\quad$ in the total absence of performance and the consequent extinction of the two populations

- $\quad$ within the limits imposed by the $K$ and $e$ components, noted upon initial reflection on each enterprise in the $Z_{2}$ population, which provide a logistical trend to $z_{2}$ with a high point that is always lower than the totality of $Z_{2}$.

High values of $z_{2}$ are conditioned by $\alpha K+\sqrt{1-\alpha^{2}} e_{i}>F^{-1}\left(P D_{i}\right)$, for which $U_{i}$ - and therefore $T L_{n}$ - tends toward zero.

\section{Conclusions}

The model's results indicate a need to make a paradigm shift in the evaluation of the creditworthiness of micro and very small enterprises. From the data collected and from the analyses carried out, current methods have proved to be ineffective in adequately supporting the productive system and, in concert with the local and national economic growth. Some points for future research seem to be:

- regulation on the incompatibility of commercial and speculative businesses in the hands of the same bank;

- strengthening studies on qualitative rating systems;

- creation of divisions, inside banks, specialized in the analysis of small economic entities with a relationship banking approach $^{17}$;

- consolidation of the cooperative credit system and credit consortiums.

The contribution offers an innovative and rigorous way - also through formalism and conceptualism of quantum mechanics - to the critical study of current rating models and their effects. The model's results indicate a need to make a paradigm shift in the evaluation of the creditworthiness of micro and very small enterprises. Stability, or rather growth, in a real economy founded on micro and very small enterprises, is a function - though not univariate - of the credit leverage disbursed. Less available credit significantly impacts the real economy, especially in countries where the financial system is centred on banks. Clearly, the rating class assigned determines whether a subject can or cannot access credit and how much it will cost. This then orients the performance results for economic micro-entities, which are structurally incapable of replacing financial resources with endogenous means, in the same terms as the Default Probability assigned by the algorithm, thus overturning the principle of cause and effect. The research tries to show how this happens. A review survey was done on national data from Italy with a comparison developed on a local subset from the Sardinia Region, as an empirical demonstration of what has been showed.

\section{Notes}

${ }^{1}$ The methodological normalization of the two databases was required, since the data from Unioncamere (Chambers of Commerce), collected during the first phase of the project, were in fact redundant. This was due to the fact that the companies, which actually closed, were not extinguished in real time due to a lack of Chamber of Commerce 
notification of their cancellation from the Register of Companies. The ISTAT data cited also had not yet been updated to the most recent periods.

${ }^{2}$ The rhythmic change occurring between 2016 and 2017 was mainly due to the sale of bank NPLs to companies specialising in recovery.

${ }^{3}$ Regardless, it is believed that not even an inverse correlation is extant: the macro enterprises appeared to be rather indifferent to the quantitative turbulence of financial leverage, at least over the short-term. Hence, the indicator shows a non-correlation, and obviously cannot be considered reliable in the perception of a negative interdependence.

${ }^{4} \mathrm{Cf}$. Panetta and Signoretti (2010).

${ }^{5}$ Cf. Conti (2016).

${ }^{6}$ Portfolio losses measured ex post with minimum offsets.

${ }^{7}$ Cf. Conti (2016).

${ }^{8}$ Cf. Fong and Vasicek (1984).

${ }^{9}$ Cf. Meyn and Tweedie (1993).

${ }^{10}$ The equation can be rewritten: $d S_{i}=S_{i}(t) r_{i} d t+S_{i}(t) \sigma_{i} d W_{i}$. Then $X_{i} \sqrt{d t}=d W_{i}$ : "Wiener process".

${ }^{11} \mathrm{Cf}$. Desogus and Casu (2018).

${ }^{12}$ The formalism and conceptualism of quantum mechanics are being borrowed for a theoretical analogy.

${ }^{13}$ The empirical demonstration of the above is work in progress. In an independent manner, an experiment was started on a sample of 100 Sardinian micro-enterprises, simulating a reversal of the rating obtained and predicting its effects on the accounting. The first tests carried out so far have shown that the denial of custody to those who have obtained it (and have continued to perform), would have led to signals of default on the $300^{\text {th }}$ day on average. Similarly, a loan to excluded micro enterprises and reaching non-performing enterprises, would have allowed a condition of regularity throughout the first year.

${ }^{14}$ A targeted analysis of the $K$ component, based on the same initial postulate, was conducted by Desogus and Casu (2019).

${ }^{15}$ The rationale for default levels could be developed in contrasting terms: the final result would be the same.

${ }^{16}$ Cf. Perko (2001).

${ }^{17}$ Cf. Fernando et al. (2002).

\section{Bibliography}

BERNANKE, B.S., GERTLER, M. and GILCHRIST, S. (1996), The Financial Accelerator and the Flight to Quality. The Review of Economics and Statistics, 78/1, 1-15.

CONTI, G. (2016). Matematica e rischio di credito. http://www.mathisintheair.org/wp.

DESOGUS, M. and CASU, E. (2018). Essays in innovative risk management methods based on deterministic, stochastic and quantum approaches. Quanah (TX): Anaphora Literary Press.

DESOGUS, M. and CASU, E. (2019). A contribution on relationship banking. Economic, anthropological and mathematical reasoning, empirical evidence from Italy. In press, forthcoming.

FERNANDO, C., CHAKRABORTY, A. and MALLIK, R. (2002). Relationship banking and credit limits. Report presented in 'Board of Governors of the Federal Reserve System', Washington D.C.

FONG, H.G. and VASICEK, O.A. (1984). A Risk Minimizing Strategy for Portfolio Immunization. The Journal of Finance, 39(5), 1541-6.

LEVINE, R., LOAYZA, N. and BECK, T. (2000). Financial intermediation and growth: causality and causes. Journal of Monetary Economics, 46(1), 31-77.

MEYN, S.P. and TWEEDIE, R.L. (1993). Markov Chains and Stochastic Stability. London: Springer.

PANETTA, F. and SIGNORETTI, F.M. (2010). Domanda e offerta di credito in Italia durante la crisi finanziaria. Bank of Italy - OccasionalPaper - Questioni di Economia e Finanza.

PERKO L. (2001). Differential Equations and Dynamical Systems. New York: Springer. 